\title{
Precursor-directed biosynthesis of new sansanmycin analogs bearing para-substituted-phenylalanines with high yields
}

\author{
Ningning Zhang ${ }^{1}$, Li Liu ${ }^{1}$, Guangzhi Shan, Qiang Cai, Xuan Lei, Bin Hong, Linzhuan Wu, Yunying Xie and \\ Ruxian Chen
}

The Journal of Antibiotics (2016) 69, 765-768; doi:10.1038/ja.2016.2; published online 24 February 2016

Sansanmycins are members of uridyl-peptide antibiotics (UPAs) isolated from Streptomyces sp. SS (CPCC 200442) in our previous researches. ${ }^{1-4}$ More than 10 related compounds have been identified, which share a common structure with pacidamycin, ${ }^{5}$ mureidomycin ${ }^{6}$ and napsamycin. ${ }^{7}$ They are composed of a $3^{\prime}$-deoxyuridyl attached via an unusual exocyclic enamide to a penta- or tetra-pseudopeptide backbone assembled by nonribosomal peptide synthetases. ${ }^{8}$ The uridyl-peptide antibiotics represent a family of promising leading compounds that they target a clinic unexplored target, MraY translocase, to inhibit the bacterial cell-wall synthesis and show good activity against highly refractory pathogens Pseudomonas aeruginosa and Mycobacterium tuberculosis. ${ }^{9}$

In our previous research, we have noted that the substrate specificity of sansanmycin biosynthesis pathway is markedly different from that of other classes of UPAs in the case of the C-terminus. ${ }^{1}$ Although all classes of UPAs can select a hydroxylphenylalanine as the C-terminus, sansanmycin chooses 4-hydroxylphenylalanine while the others choose 3-hydroxylphenylalanine (Figure 1). We were therefore curious about whether this difference in substrate specificity could lead to new sansanmycin analogs by precursor-directed biosynthesis, a simple but effective method for generating modified natural products in the producing organism itself. So five phenylalanine derivatives (4-F-, 4$\mathrm{Cl}-$, 4-Br-, 4-Me- and 4-MeO-phenylalanines) were picked out and tested for their incorporation, which led to four new sansanmycin analogs. Here we report on their precursor-directed biosynthesis, isolation, structure elucidation and antibacterial activity.

Streptomyces sp. SS. was cultivated on an ISPII slant containing yeast extract $0.4 \%$, glucose $0.4 \%$, malt extract $1 \%$, agar $1.5 \%$ at $28{ }^{\circ} \mathrm{C}$ for 7 days, then inoculated into the sansanmycin production medium, consisting of glucose $3 \%$, starch $0.5 \%$, peptone $0.6 \%$, $\left(\mathrm{NH}_{4}\right)_{2} \mathrm{SO}_{4} 0.7 \%$, and $\mathrm{CaCO}_{3} 0.2 \%$, and incubated at $28{ }^{\circ} \mathrm{C}, 180 \mathrm{r}$. p.m. for $48 \mathrm{~h}$. The obtained preculture was further inoculated into the sansanmycin production medium supplementary with the selected phenylalanine derivatives at a final concentration of $3 \mathrm{~mm}$ and incubated for 5 days. The cultures were enriched by Sep-Pak $\mathrm{C}_{18}$ Cartridges (WATERS, Milford, MA, USA) and then analyzed by liquid chromatography-mass/mass spectra for the presence of the expected products. Liquid chormatography-mass spectra was conducted on an Agilent 1200 instrument (Agilent Technologies, Santa Clara, CA, USA) coupled to a Finnigan LTQ $\mathrm{XT}$ ion trap mass spectrometer (Thermo Fisher Scientific, Waltham, MA, USA) by using negative electrospray ionization and an XBridge $\mathrm{C}_{18}$ column (Waters; $4.6 \times 150 \mathrm{~mm}, 3.5 \mu \mathrm{m}$, solvent A: $\left.0.1 \%(\mathrm{w} / \mathrm{v})\left(\mathrm{NH}_{4}\right)_{2} \mathrm{CO}_{3}\right)$, solvent $\mathrm{B}$ : $\mathrm{MeOH}$; flow rate, $1 \mathrm{ml} \mathrm{min}^{-1}$; $0-40 \mathrm{~min}, 20-45 \% \mathrm{~B}$ (linear gradient), $40-50 \mathrm{~min}, 45 \% \mathrm{~B}$ ). The results indicated that four precursors (4-Cl-, 4-Br-, 4-Me- and 4$\mathrm{MeO}-$ phenylalanines) were incorporated into the C-terminus of the peptide backbone of sansanmycin to generate corresponding analogs at higher levels than sansanmycin $\mathrm{H}$ (Figure 2). Especially, the yield of [4-Me-Phe]-sansanmycin $\mathrm{H}$ was $\sim 19$ times more than that of sansanmycin H. Then, to access enough amount of material for further structure elucidation and bioassay, fermentations with these four precursors were scaled up to volumes ranging from $2-51$ according to the yields of the corresponding products. The expected sansanmycin analogs were isolated and purified according to the reported method with minor modifications. ${ }^{1}$ Briefly, the sansanmycin analogs were enriched from the fermentation broth by macroporous absorbent resin 4006 column. The obtained crude was subsequently applied on Toyopearl DEAE $650 \mathrm{~m}$ column and eluted with Tris- $\mathrm{HCl}(20 \mathrm{mM}, \mathrm{pH} 8.5)$ plus $\mathrm{NaCl}$ and monitored by ultra-violet to yield expected products. Finally, the obtained crude products were further purified using high performance liquid chromatography (HPLC) (YMC-Pack ODS-A $5 \mu \mathrm{m}, 250 \times 20 \mathrm{~mm}$

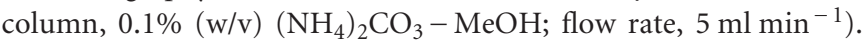

Institute of Medicinal Biotechnology, Chinese Academy of Medical Sciences \& Peking Union Medical College, Beijing, China

${ }^{1}$ These authors contributed equally to this work.

Correspondence: Dr Y Xie or Professor R Chen, Institute of Medicinal Biotechnology, Chinese Academy of Medical Sciences \& Peking Union Medical College, Tiantanxili No.1, Beijing, China.

E-mail: xieyy@imb.pumc.edu.cn or chenrx888@sohu.com

Received 2 November 2015; revised 23 December 2015; accepted 5 January 2016; published online 24 February 2016 
The yields of [4-R-Phe]-sansanmycin $\mathrm{H}(\mathrm{R}=\mathrm{Cl}, \mathrm{Br}, \mathrm{Me}, \mathrm{MeO})$ were $2.0,1.9,6.2$ and $1.7 \mathrm{mgl}^{-1}$, respectively. Nuclear magnetic resonace (NMR) data of these purified compounds were obtained with Varian Mercury 600 spectrometers (Varian, Palo Alto, CA, USA) using $\mathrm{D}_{2} \mathrm{O}(\mathrm{pD}=8.0)$ or dimethylsulfoxide (DMSO) as solvent.

[4-Me-Phe]-sansanmycin $\mathrm{H}(\mathbf{1})$ is a white powder (melting point $204-206^{\circ} \mathrm{C}$ ) with a molecular weight of 838 , two mass units smaller than that of sansanmycin $\mathrm{H}$, which hinted at the replacement of the hydroxyl group in sansanmycin $\mathrm{H}$ with a methyl group in compound

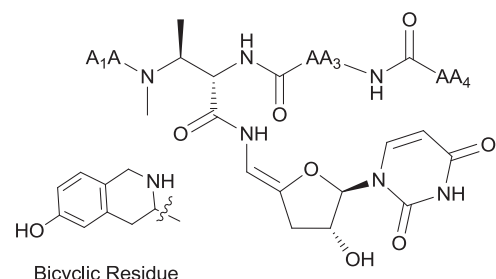

\begin{tabular}{llll}
\hline & AA1 & AA3 & AA4 \\
\hline Sansanmycin A & $m$-Tyr & Met & Trp \\
Sansanmycin H & $m$-Tyr & Met & Tyr \\
Mureidomycins & $m$-Tyr & Met & $m$-Tyr \\
Pacidamycins & $m$-Tyr/Ala & Ala & Trp/Phe/m-Tyr \\
Napsamycins & Bicyclic Residue & Met & $m$-Tyr \\
$\mathbf{1}$ & $m$-Tyr & Met & 4-Me-Phe \\
$\mathbf{2}$ & $m$-Tyr & Met & 4-Cl-Phe \\
$\mathbf{3}$ & $m$-Tyr & Met & 4-Br-Phe \\
$\mathbf{4}$ & $m$-Tyr & Met & 4-MeO-Phe \\
\hline
\end{tabular}

Figure 1 Structures of uridyl-peptide antibiotics.
1. Furthermore, the daughter ion $m / z 658$, corresponding to the loss of C-terminal amino acid of sansanmycin $\mathrm{H}$, also appeared as base peak in the electrospray ionization mass/mass spectrum of compound $\mathbf{1}$, which indicated the 4-methylphenylalanine was incorporated into the C-terminus of 1 . Compared with the ${ }^{1} \mathrm{H}$ NMR spectrum of sansanmycin $\mathrm{H}^{1}{ }^{1}$ that of 1 showed an extra methyl proton signal at $\delta 2.29\left(\mathrm{~s}, \mathrm{Ar}-\mathrm{CH}_{3}\right)$, which further confirmed the above hypothesis. Interpretation of the 2D NMR and ESIMS/MS data (Table 1 and Figure 2) also confirmed this expected structure.

[4-Cl-Phe]- (2), [4-Br-Phe]- (3) and [4-MeO-Phe]-sansanmycin $\mathrm{H}$ (4) have expected molecular weights of 858, 902 and 854, respectively. Moreover, the ESIMS/MS of all three compounds showed a diagnostic daughter ion, $m / z$ 658, for the loss of the C-terminal amino acid, and the ESIMS of $\mathbf{3}$ and $\mathbf{4}$ exhibited characteristic isotope patterns of chlorinated and brominated sansanmycin $\mathrm{H}$, separately. All of these indicated that the precursors added were incorporated into the C-terminal part of sansanmycin. Interpretation of the NMR and ESIMS/MS data (Table 1 and Figure 2) further identified these compounds as expected structures.

The antibacterial activities of compounds (1-4) were assayed and listed in Table 2 in comparison to sansanmycin $\mathrm{H}$, which served as a reference. While the majority of obtained compounds performed as well as or worse than their natural parent, sansanmycin $H$, one noteworthy improvement from the suite of sansanmycin $\mathrm{H}$ derivatives was identified; both [4-Br-Phe]- and [4-Cl-Phe]-sansanmycin $\mathrm{H}$ exhibited fourfold increase in potency over sansanmycin $\mathrm{H}$ against Staphylococcus aureus (the minimal inhibitory concentration (MIC) $32 \mathrm{vs} 128 \mu \mathrm{g} \mathrm{ml}^{-1}$ ). This improvement might be explained by the fact that both [4-Br-Phe]- $(\log P=1.08)$ and [4-Cl-Phe]- $(\log P=0.91)$ sansanmycin $\mathrm{H}$ had higher lipophilicity than their parent (log $P=-0.42$ ), which could lead to an increase of passive diffusion through the cytoplasmic membrane of $S$. aureus. Another notable observation was that all tested compounds were potential against $E$. coli $\Delta$ tolC mutant $\left(\mathrm{MIC}=1-4 \mu \mathrm{g} \mathrm{ml}^{-1}\right)$ while inactive against

a

b

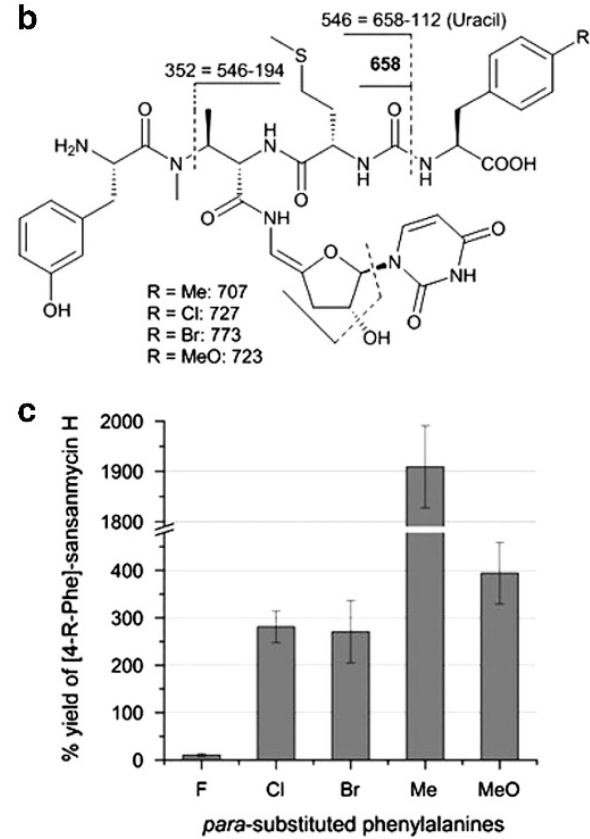

Figure 2 Extracted-ion chromatograms (a) and MS/MS analyzes (b) of compounds (1-4). (c) The yields of [4-R-Phe]-sansanmycin H relative to sansanmycin $\mathrm{H}$ formed in the control. Experiments were carried out in triplicate with standard deviation indicated by error bars. 


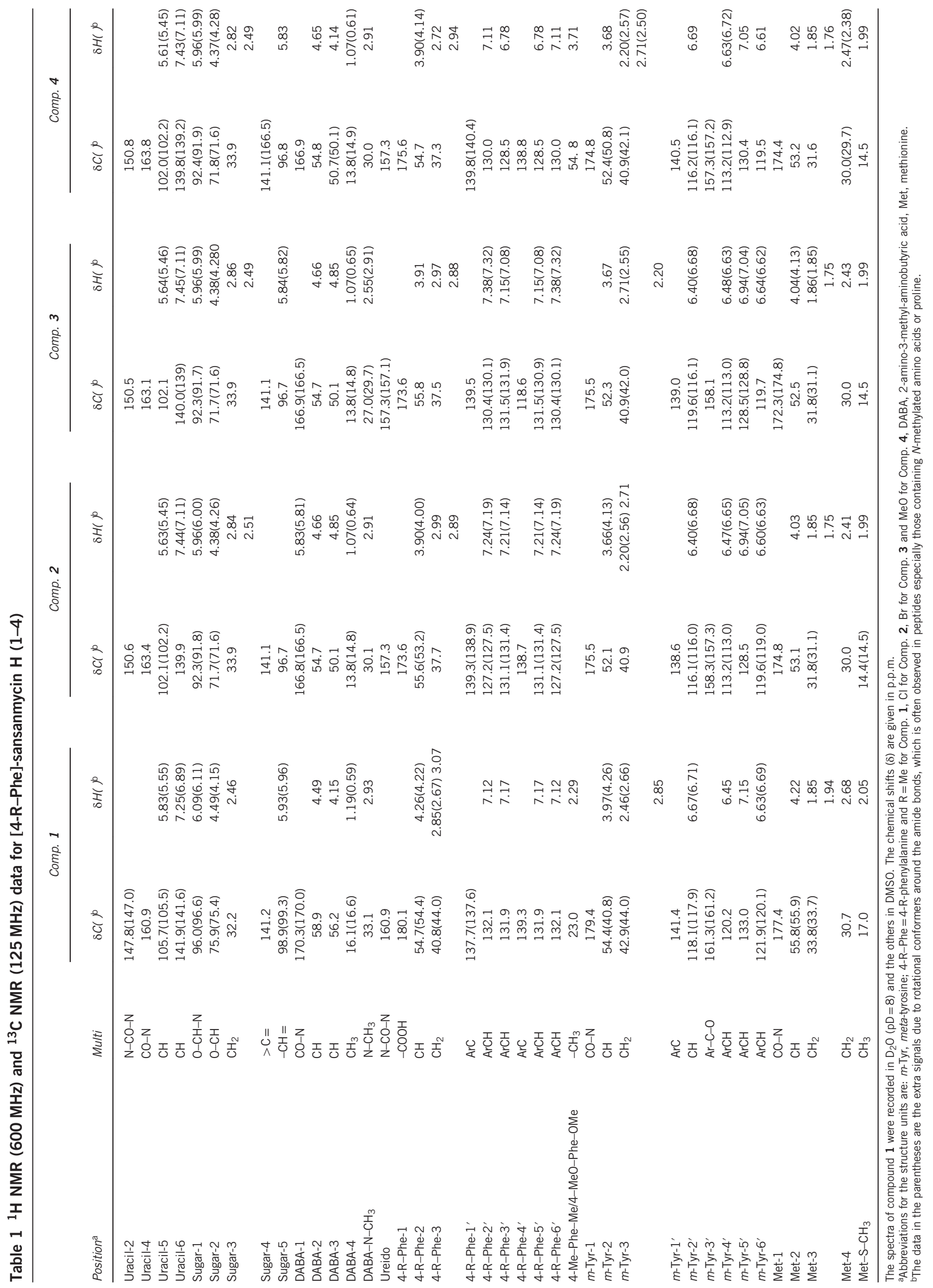


Table 2 Activity of precursor-directed biosynthetic sansanmycin analogs<smiles>[R]c1ccc(C[C@H](NC(=O)N[C@@H](CCSC)C(=O)N[C@H](C(=O)N/C=C2/C[C@H](O)[C@H](n3ccc(=O)[nH]c3=O)O2)[C@H](C)N(C)C(=O)[C@H](N)Cc2cccc(O)c2)C(=O)O)cc1</smiles>

\begin{tabular}{|c|c|c|c|c|c|c|c|}
\hline \multirow[b]{3}{*}{ Compd } & \multirow[b]{3}{*}{$R$} & \multirow[b]{3}{*}{$\log P^{a}$} & \multicolumn{5}{|c|}{$M I C\left(\mu g m l^{-1}\right)$} \\
\hline & & & \multirow{2}{*}{$P A^{\mathrm{b}}$} & \multirow{2}{*}{$S A^{\mathrm{b}}$} & \multirow{2}{*}{$M S^{b}$} & \multicolumn{2}{|c|}{ E. coli } \\
\hline & & & & & & Wild type & $\Delta$ tolC mutant \\
\hline 1 & $\mathrm{Me}$ & 0.77 & 16 & 64 & $>128$ & $>128$ & 2 \\
\hline 2 & $\mathrm{Cl}$ & 0.91 & 16 & 32 & $>128$ & $>128$ & 1 \\
\hline 3 & $\mathrm{Br}$ & 1.08 & 16 & 32 & $>128$ & $>128$ & 1 \\
\hline 4 & $\mathrm{MeO}$ & 0.23 & 32 & 128 & $>128$ & $>128$ & 4 \\
\hline SSH & $\mathrm{OH}$ & -0.42 & 4 & 128 & 64 & $>128$ & 1 \\
\hline
\end{tabular}

${ }^{a}$ Log $P$ values were calculated by ACD/ChemSketch software (12.0); Advanced Chemistry Development, Inc., Toronto, ON, Canada.

PPA, P. aeruginosa 11 (wild type); SA, S. aureus CPCC100051 (wild type); MS, M. smegmatis CPCC240556 (wild type).

wild-type E. coli $\left(\mathrm{MIC}>64 \mu \mathrm{g} \mathrm{ml}^{-1}\right.$ ). This is the same as the situation of mureidomycin $\mathrm{A}$ and $\mathrm{C}$, in which the expression of multidrug efflux system AcrAB-TolC was proved to mediate the intrinsic resistance of E. coli to mureidomycin. ${ }^{10}$ All of these results hinted that the uptake and the efflux might be limiting factors in the antibacterial activities of sansanmycin.

\section{CONFLICT OF INTEREST}

The authors declare no conflict of interest.

\section{ACKNOWLEDGEMENTS}

We thank Dr Bertolt Gust (Pharmaceutical Institute, University of Tuebingen, Germany) for kindly providing Escherichia coli $\Delta t o l C$ mutant strain. We also acknowledge financial support from the National Natural Science Foundation of China (81273415 and 81402836) and National Mega-Project for Innovative Drugs (2012ZX09301002-001-018).

1 Xie, $Y$. et al. NRPS substrate promiscuity leads to more potent antitubercular sansanmycin analogues. J. Nat. Prod. 77, 1744-1748 (2014).

2 Xie, Y., Chen, R., Si, S., Sun, C. \& Xu, H. A new nucleosidyl-peptide antibiotic, sansanmycin. J. Antibiot. (Tokyo) 60, 158-161 (2007).

3 Xie, Y., Xu, H., Si, S., Sun, C. \& Chen, R. Sansanmycins B and C, new components of sansanmycins. J. Antibiot. (Tokyo) 61, 237-240 (2008).

4 Xie, Y., Xu, H., Sun, C., Yu, Y. \& Chen, R. Two novel nucleosidyl-peptide antibiotics: Sansanmycin F and G produced by Streptomyces sp SS. J. Antibiot. (Tokyo) 63, 143-146 (2010).

5 Chen, R. H., Buko, A. M., Whittern, D. N. \& McAlpine, J. B. Pacidamycins, a novel series of antibiotics with anti-Pseudomonas aeruginosa activity. II. Isolation and structural elucidation. J. Antibiot. (Tokyo) 42, 512-520 (1989).

6 Isono, F. et al. Mureidomycins A-D, novel peptidylnucleoside antibiotics with spheroplast forming activity. II. Structural elucidation. J. Antibiot. (Tokyo) 42, 667-673 (1989)

7 Chatterjee, S. et al. Napsamycins, new Pseudomonas active antibiotics of the mureidomycin family from Streptomyces sp. HIL Y-82,11372. J. Antibiot. (Tokyo) 47, 595-598 (1994).

8 Zhang, W., Ostash, B. \& Walsh, C. T. Identification of the biosynthetic gene cluster for the pacidamycin group of peptidyl nucleoside antibiotics. Proc. Natl Acad. Sci. USA 107, 16828-16833 (2010).

9 Winn, M., Goss, R. J., Kimura, K. \& Bugg, T. D. Antimicrobial nucleoside antibiotics targeting cell wall assembly: recent advances in structure-function studies and nucleoside biosynthesis. Nat. Prod. Rep. 27, 279-304 (2010).

10 Gotoh, N. et al. Intrinsic resistance of Escherichia coli to mureidomycin A and C due to expression of the multidrug efflux system AcrAB-TolC: comparison with the efflux systems of mureidomycin-susceptible Pseudomonas aeruginosa. J. Infect. Chemother. 9, 101-103 (2003).

Supplementary Information accompanies the paper on The Journal of Antibiotics website (http://www.nature.com/ja) 